\title{
Medicina Interna y Salud Pública: Claves para una relación virtuosa
}

\author{
Grupo de Estudios en Medicina Interna y Salud Pública, \\ Sociedad Médica de Santiago - \\ Sociedad Chilena de Medicina Interna ${ }^{1}$.
}

\section{Internal Medicine and Public Health}

\begin{abstract}
A special Committee on Internal Medicine and Public Health was established by Sociedad Médica de Santiago (Chilean Society of Internal Medicine) in April 2007 with the duty to write a Consensus Paper on the interaction between both branches of medical profession. The main objective was to find the common grounds on which to construct a positive approach to regain space for Internal Medicine, based on prevalent epidemiological features related to adult health issues. The authors describe the reasons to explain the gap between clinical medicine and population health and identify the nature and evolution of chronic diseases as the point of encounter between both. With Chilean health surveys data, they state that chronic diseases explain the high proportion of burden of disease, mortality and disability, and stress that by the year 2025 one in every five inhabitants will be over 65 years of age, with ageing as another main problem for the health care sector. Population with multiple risks and multimorbidity is the most important challenge for the Chilean Health Care System. A new model of care is needed to tackle this scenario with new skills regarding psychosocial determinants of health. The leading role of internists and ideally geriatricians, will be crucial in this process and will help the implementation of sound population based interventions. Both individual and community level interventions will help to improve quality of life of Chilean families (Rev Méd Chile 2009; 137: 1095-8).
\end{abstract}

(Key words: Delivery of Health Care; Internal Medicine; Public Health)

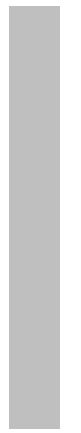

\footnotetext{
${ }^{1}$ Miembros del Comité y autores del documento son los Dres. Jorge Jiménez de la Jara (Presidente del Grupo, Departamento de Salud Pública, Pontificia Universidad Católica de Chile), Cecilia Albala (Instituto de Nutrición y Tecnología de Alimentos, Universidad de Chile), Claudia Bambs (Departamento de Salud Pública, Pontificia Universidad Católica de Chile), Edgardo Cruz Mena (Departamento de Enfermedades Respiratorias, Facultad de Medicina, Pontificia Universidad Católica de Chile), Manuel Espinoza (Departamento de Salud Pública, Pontificia Universidad Católica de Chile), Paula Margozzini (Departamento de Salud Pública, Pontificia Universidad Católica de Chile), Gabriel Rada (Unidad Docente Asociada HSR, Departamento de Medicina Interna y Programa de Salud Basada en Evidencia, Pontificia Universidad Católica de Chile) y Pedro Paulo Marín (Centro de Geriatría y Gerontología, Departamento de Medicina Interna, Pontificia Universidad Católica de Chile, Presidente de la Sociedad Médica de Santiago).
}

Correspondencia a: Dr. Jorge Jiménez de la Jara. E mail: jjimenez@med.puc.cl 
$\mathrm{E}^{n}$ n abril de 2008 se constituyó el Grupo de Estudios en Medicina Interna y Salud Pública de la Sociedad Médica de Santiago - Sociedad Chilena de Medicina Interna. El propósito de este grupo ha sido reflexionar sobre la realidad de la salud en Chile y, específicamente, contribuir a la discusión sobre la necesaria asociación entre la Medicina Interna y la Salud Pública. El presente documento contiene una síntesis de los principales temas abordados y ha sido redactado con el propósito de estimular su discusión por la comunidad médica.

La Salud Pública y la Medicina Interna, dos de las especialidades más tradicionales de la Medicina, en las últimas décadas se han desarrollado por caminos separados, casi opuestos. Esta diferenciación parece haberse profundizado al punto de visualizar al internista y al salubrista como dos extremos del continuo de la atención médica. Por un lado, el médico internista tendría un rol predominantemente asistencial, hospitalario, enfocado en el tratamiento agudo de la enfermedad. Por el otro, el salubrista, estaría enfocado en el abordaje poblacional de problemas de alta prevalencia, con énfasis en la prevención, pero sin contacto directo con el paciente. Sin embargo, ambos comparten la aspiración de contribuir al mayor grado de salud posible para las personas y las poblaciones que ellas conforman. Este objetivo muy difícilmente será logrado sin un diálogo entre ambas disciplinas.

Por otra parte, los modelos de atención médica han ido cambiando enormemente desde mediados del siglo pasado, creando varios efectos en la distribución de tareas con respecto a la prevención y manejo de las enfermedades, a lo que se agrega un incremento sustancial de la complejidad y costos de la medicina. Estos cambios han provocado desorientación y pérdida de claridad en el ejercicio de la profesión y se ha hablado de la "infelicidad" o "malestar de los médicos" como una forma de graficar su inserción en una sociedad que exige resultados pero, simultáneamente, arremete contra los profesionales cuando no ve satisfechas sus expectativas ${ }^{1}$.

ENFERMEDADES CRÓNICAS:

Encuentro entre Medicina Interna y Salud Pública

La transformación epidemiológica que ha sufrido Chile hacia un predominio de enfermedades crónicas, constituye un punto natural de encuentro entre estas dos disciplinas.

Los problemas de salud del adulto son hoy los que más influyen sobre los indicadores del nivel de salud de la población chilena ${ }^{2}$ y las enfermedades crónicas explican la mayor parte de la carga de enfermedad, muerte y discapacidad en Chile $^{3}$. Actualmente, uno de cada diez chilenos son adultos mayores y para el año 2025 esta proporción aumentará a una de cada cinco personas. Este cambio etario genera la obligación de implementar políticas e intervenciones para prevenir el desarrollo de enfermedades crónicas que lleven a la dependencia funcional del adulto mayor, principal fuente de sufrimiento personal y costo social del envejecimiento ${ }^{4}$.

Las enfermedades crónicas están estrechamente relacionadas tanto con el envejecimiento poblacional como con la adopción de estilos de vida no saludables. Es así como, por ejemplo, el estilo de consumo de alcohol en Chile es la conducta de riesgo que hoy produce una mayor carga de muerte y discapacidad en nuestro país, aún más que la forma en que comemos, el sedentarismo, e incluso el tabaquismo. Detrás de estas conductas y estilos de vida se encuentran fuertes determinantes psicológicas y sociales que no podemos desconocer: nivel socioeconómico y educacional, ambiente familiar, "marketing", leyes. En fin, todo el marco social en el cual las personas crecen y se desarrollan.

Desde el punto de vista clínico, la prevención y tratamiento de condiciones tan prevalentes como la depresión, la hipertensión, la obesidad, el tabaquismo y el consumo excesivo de alcohol son ejemplos de problemas de salud complejos que requieren la integración de equipos interdisciplinarios, cuya acción debe ser capaz de impactar al paciente y a su contexto. De otra forma, los esfuerzos de cambio individual son difícilmente sostenibles en el tiempo. Todo esto genera un flujo de influencias saludables desde el paciente individual hacia su entorno, contribuyendo a fortalecer las acciones que avanzan lentamente desde la Salud Pública. En este punto, es importante que tanto internistas como salubristas confíen en que estos cambios sí son posibles, tanto a nivel individual como poblacional. Se requiere que ambos actores aúnen esfuerzos de liderazgo en la búsqueda de la mejor evidencia y en la 
abogacía para que las acciones de Salud Pública puedan encontrar apoyo, tanto a nivel de los pacientes y sus familias, como también a nivel de los tomadores de decisión.

\section{Multimorbilidad: EL VERDADERO DESAFÍO}

Una mirada integradora entre la Medicina Interna y la Salud Pública debe ir más allá del abordaje artificial de los riesgos por separado, y exige la consideración de un escenario real, el de los "riesgos múltiples", riesgos que tienen un efecto amplificador entre sí, multiplicando más que sumando el riesgo global para los individuos afectados. Un ejemplo de esto es la combinación de tabaquismo, sedentarismo y sobrepeso-obesidad, que se encuentra presente en el $20 \%$ de la población chilena entre 25 y 44 años $^{5}$, y que aumenta más de cinco veces el riesgo de desarrollar una patología vascular entre los 50 y 65 años $^{6}$. Por otra parte, no sólo los riesgos son múltiples y coexistentes: también lo son las enfermedades crónicas causadas por estos riesgos. Encontrar a un adulto chileno sin enfermedades crónicas es una rareza (menos de $11 \%$ de los adultos); más aún, $66 \%$ de la población chilena adulta vive con al menos dos enfermedades crónicas y existe 10\% (un millón y medio de personas) que vive con al menos 5 enfermedades crónicas simultáneas. Esta multimorbilidad afecta la calidad de vida y es responsable de un gran aumento de los costos para el sistema de salud ${ }^{7}$. Por otra parte, la mayoría de estas personas se encuentran en edad laboralmente activa (86\% son menores de 65 años) y casi la mitad de ellos tienen menos de 8 años de educación ${ }^{8}$, lo que refleja la distribución desigual de este tipo de enfermedades, en desmedro de la población que dispone de menos recursos en Chile.

\section{NueVaS Habilidades PaRa Un NUEVO MODELO} DE ATENCión: Desafío Para internistas y SALUbristas

El perfil epidemiológico del país orienta hacia un grupo de patologías y factores de riesgo relacionado principalmente con problemas neuropsiquiátricos, digestivos, cardiovasculares y músculo-esqueléticos, todos con alta prevalencia. La magnitud de estos problemas hace que sean inabordables por el recurso médico especialista. Es decir, es necesario el protagonismo de médicos "generalistas" (internistas, geriatras, médicos generales y familiares) que sean capaces de trabajar interdisciplinariamente y en red y, además, puedan generar iniciativas que vuelvan a posicionar al paciente junto a su núcleo familiar y comunitario en el centro de la toma de decisiones en salud, permitiendo así privilegiar el manejo integral de los individuos con múltiples condiciones de riesgo.

En este escenario preventivo, la Medicina Interna puede hacer una enorme contribución siempre que se potencie la integración con otras especialidades, entre los distintos niveles de atención (internistas, geriatras y subespecialistas hospitalarios, atención primaria y medicina familiar) y se promueva el desarrollo del enorme potencial de los otros integrantes del equipo de salud (enfermeras, auxiliares, nutricionistas, psicólogos, agentes comunitarios, etc.).

Finalmente, interesa destacar un contraste entre los alcances de la acción del médico ante las enfermedades agudas, en comparación con las enfermedades crónicas. En el enfrentamiento de las primeras, el médico realiza una acción que se enmarca en un tiempo limitado, cuyos efectos son duraderos (el paciente se cura de la enfermedad) $\mathrm{y}$ dependen en buena medida de las decisiones diagnósticas y terapéuticas del médico. Por el contrario, en una enfermedad crónica, el contacto entre el internista y la historia natural de la enfermedad de su paciente es muy breve, constituye una proporción muy pequeña del total y la efectividad de la intervención va a depender mayormente de sus habilidades psicosociales y comunicacionales (habilidades para motivar el cambio de conducta y adherencia), de la forma en que el modelo de atención facilita las cosas a los pacientes y de la forma en que las políticas de salud y las políticas públicas, en general, incentivan y apoyan los esfuerzos de cambio. La definición clara de estos alcances puede contribuir a la optimización del rol del médico internista en un contexto ambulatorio, su coordinación con el resto del equipo de salud y la necesidad de generar alianzas con el paciente y su núcleo, de modo de promover el autocuidado y trabajar las estrategias y apoyos adecuados para que el paciente conduzca su enfermedad en forma prota- 
gónica y asuma la responsabilidad y el desafío de mantenerse en el mejor estado de salud posible.

En suma, mientras las medidas de Salud Pública toman tiempo en implementarse y en ese proceso deben enfrentar una multiplicidad de barreras, los equipos clínicos y, especialmente los médicos internistas y sus equipos, son llamados a contribuir activamente al enfrentamiento de los problemas de salud que están menguando hoy la calidad de vida de la población chilena. La contribución del internista se vislumbra fundamentalmente desde el ámbito clínico individual,

\section{REFERENCIAS}

1. Jiménez J. Cambio en el entorno del trabajo médico. Rev Méd Chile 2004; 132: 637-42.

2. Giusti A, Arteaga O, Bedregal P, Margozzini P, Sotelo JM. Chile. En: Health in the Americas 2007. Scientific and Technical Publication № 622, Washington DC, PAHO 2007 (ISBN 9789275116229).

3. MINSAL Estudio de carga de enfermedad y carga atribuible, Chile 2007. En: http//www.minsal.cl

4. Marín PP. Reflexiones para considerar en una política pública de salud para las personas mayores. Rev Méd Chile 2007; 135: 392-8.

5. MargozZini P. Obesidad y multimorbilidad por enfermedades crónicas en Chile. En: Mardones F (Ed.) Obesidad en Chile ¿Qué podemos hacer? Ediciones sin embargo, debe empoderarse y enfatizar su rol como líder de opinión dentro de la comunidad médica y la población en general, así como su aporte al desarrollo e implementación de programas y políticas de salud. Se necesita la participación e interacción efectiva de todos los actores relevantes para que las conductas y estilos de vida saludables se conviertan en verdaderas inquietudes ciudadanas, tan legítimas y exigibles como las preocupaciones ecológicas y ambientales. La Medicina Interna y la Salud Pública no pueden restarse de estos desafíos.
Universitarias P. Universidad Católica de Chile, Santiago, 2009.

6. Wannamethee SG, Shaper AG, Walker M, Ebrahim $S$. Lifestyle and 15 year survival free of heart attack, stroke and diabetes en middle aged British men. Arch Intern Med 1998; 158: 2433-40.

7. Fortín M, Lapointe L, Hudon C, Vanase A, Ntetu AL, MaLtais D. Multimorbidity and quality of life in primary care: a systematic review. Health and Quality of Life Outcomes 2004; 2: 51.

8. Margozzini P, Olea R (2005). Multimorbilidad y comorbilidad por enfermedades crónicas del adulto en Chile: resultados de la Primera Encuesta Nacional de Salud - 2003. Informe III del proyecto MINSAL/ OPS “Análisis en profundidad de la ENS2003". 